\title{
Advanced behavioural screening: automated home cage ethology
}

\author{
Berry M. Spruijt ${ }^{1,2}$, Leonie DeVisser ${ }^{1,2, *}$ \\ 'Department of Animals, Science and Society, Utrecht University, Yalelaan 2, 3584 CM Utrecht, The Netherlands \\ ${ }^{2}$ Rudolf Magnus Institute of Neurosciences, UMC Utrecht, The Netherlands
}

\begin{abstract}
Animal behaviour has been studied using two approaches, (I) well-controlled experiments focusing on specific responses and (2) those with natural - fuzzy - but biologically relevant conditions. Ideally, one behavioural test should be able to address both. The home cage provided with various stimuli is proposed as an allin-one possibility. This, however, results in an exponential increase in complexity regarding observation and analysis tools. It seems difficult to accept that behavioural expressions need a mathematical approach to unravel its organisation and meaning. Developments in artificial intelligence and data mining are essential to accelerate this necessary evolution in behavioural sciences.
\end{abstract}

\section{Introduction}

It is commonly accepted and has been described before; behaviour is the ultimate and most complex output of the brain. The complexity and flexibility of behaviour idiosyncratic to the adaptive capacity of an organism is realized by environmental influences on gene expression as well as the genetic predisposition for such influences. Genes are embedded in hierarchically organized neuronal networks, which underlie to a great extent brain plasticity. Pertinent examples of animal models used in behavioural genetics showing the consequences of long-term environmental influences have been reviewed elegantly by McClearn [1]. He

*Corresponding author: L. DeVisser (I.devisser@vet.uu.nl)

\section{Section Editors:}

Bart Ellenbroek - Radboud University, Nijmegen, The Netherlands

Twan Ederveen - N.V. Organon, Oss, The Netherlands

provides examples of studies in the 50s and 60s, which repeatedly reported that behavioural performance of rats and mice in tests designed to determine gene or drug effects, strongly depend on gender, age and a large variety of characteristics of the test and housing conditions. For instance, it was shown that in rats selected for performance in a cognition test, so called 'Bright' and 'Dull' animals, their performance was affected by environmental enrichment, age and gender [2].

Recently, the complexity and pitfalls in behavioural neuroscience received attention again [3-6], not in the least due to the need of reliable and high-throughput behavioural assessments for the elucidation of the functional role of genes in behaviour. Würbel [3] argues that the answer as often sought in standardization and exhaustive listing of all possible factors involved, is inefficient and in fact misleading. He pleads for a naturalistic, thus enriched, test environment reducing between-laboratory variance allowing biologically relevant testing of mutant mice [7]. One other complicating factor is individual variation in genetically identical animals. The role of complex social structures and dominance status are the result of early genetic and environmental influences that programme behaviour of later life. Such variability should not be eliminated by standardization but be subject of study itself [8].

Thus, the long recognized problem of reliability and functional relevance of behavioural tests is not simply a matter of 
better testing or more standardization alone: It requires a thorough analysis of the approaches underlying the behavioural tests.

\section{Two types of approaches for two types of questions}

When drugs or gene manipulations are tested on their efficacy in modifying animal or human behaviour, two types of questions have to be distinguished. (1) To what extent is the intervention causing a functional or biologically relevant effect? For this question tests have been developed to address these specific functional aspects. If we take the example of anxiety, a common characteristic of these functional tests is the similarity of the animals' reaction with the natural defensive or safety seeking response. For instance, rodents are exposed to an alarm call emitted by a bird alerted by a predator [9] or tested in the Mouse Defense Test Battery [10].

(2) To what extent has a gene or drug manipulation a specific effect on a one-dimensional parameter? For this type of question, tests have been developed that either applied simplified forms of functional tests or make use of responses that not necessarily have a functional meaning. For example, the 'small open field' [11] is legitimately used for assessing dopaminergic activity as measured by horizontal and vertical locomotor activity but does not allow a functional interpretation.

Now these two types of approaches, both valid, but aimed at different type of research questions, required almost totally different testing rationales. The above-mentioned different approaches with their respective underlying rationales have been reviewed by Tecott and Nestler [12]. Behavioural pharmacology adopted the functional test, but used and reduced them more and more for their specific questions ignoring the context and history of the animals in favour of efficiency. In case of anxiety, light-dark gradients or open spaces, such as the open field test, elevated plus maze or light-dark box (reviewed by Crawley [13]) are widely adopted. When these tests coincide with a short observation time and a limited number of responses, dissection of multiple factors underlying anxiety is hardly possible. The emphasis, methodologically, moved towards the conditions within the test and assumed standardization of conditions outside the test arena or at least underestimated the influence of external conditions.

The pharmacological validation of the test - once an accepted drug confirmed the biological expectation replaced functional testing. The efficacy of a reference drug allowed the divergence of standard tests; as long as benzodiazepines are effective it seems justified to label the response as an anxious response assuming that the efficacy of benzodiazepines is the golden standard for anxiety. Eventually, we face reports on nocturnal animals being tested for a short time period in bright light situations in stimulus-poor tests in separate experimental rooms with little attention for their living conditions outside and preceding this test period.

\section{Methodological limitations}

Because the relative difference between the experimentally treated animals and their control groups are supposed to be decisive, differences between the tests set-ups and the conditions outside the tests diverged. In fact, so-called standard open fields, elevated plus mazes, light-dark boxes and burying tasks are nowhere the same. The conditions of animals related to ontogeny and procedures around testing are hardly discussed. If again anxiety is considered, discrepancies in for instance inbred strain rankings are easy to find. Results vary from BALB/c being more anxious than C57BL/6 [14,15] no differences [16] or C57BL/6 displaying more anxiety-related behaviour than BALB/c $[17,18]$. In addition, sensitivity to diazepam as an anxiolytic compound differs between laboratories and tests $[15,19]$. Different tests appear to measure different aspects of anxiety, pleading for the integration of tests to catch a comprehensive and reliable anxiety profile of an animal.

In addition to this there is the scoring of behaviour by a human observer; contents and use of an ethogram is also far form standardized. In fact even within the same laboratory using the same ethogram $80 \%$ reliability between observers is often satisfactory. Large-scale comparisons across laboratories show poor reproducibility, in particular for tests of locomotor and exploratory activity [20,21]. In absence of the pharmacological validation of a reference drug the test itself appeared to be a poor instrument. For instance CA1-specific NMDA receptor-knockout mice having a learning deficit in a novel-object recognition task did not show any deficit when housed in an enriched environment [22].

In principle, the caveats reappearing in behavioural genetics are not different from those previously met in drug screening and have led to the discipline ethopharmacology. Ethopharmacology tries to combine ethological rationales in behavioural pharmacology. It introduced the use of rather complex test situations such as resident-intruder models for studying agonistic behaviour [23,24] as a model for depression and chronic stress and a much more elaborate analysis of existing tests such as elevated plus maze [25]. Increased complexity in the test situation also meets another indispensable condition: the activation of more behavioural motivational systems interacting with each other. However, intensive labour necessary for conducting complicated tests and the time required to train human observers prevented a general use of the implementation of ethological methods. Now, automation as a means to enhance efficiency is an ongoing and well-known instrument. But the mere automation alone of already existing tests is not the optimal answer to generate efficient tests providing qualitative and quantitative information on behaviour. The question is: how can implicit or explicit strategies underlying the observation and analysis of animal behaviour be best automated avoiding that 
conceptual limitations of commonly used tests are automatically incorporated as well?

\section{A complex home cage as test environment}

The ultimate test of course requires having it all in one, both qualitative and quantitative aspects of spontaneous and acquired behaviours. The nature of the 'ideal' test and its technical features can be deduced from the functional requirements listed in Table 1.

An important source of confounding variables and waste of potential information is formed by neglecting basal behaviour in the standard housing cage and transporting the animals with its accompanying stress to a novel test cage and back. In addition, it has been reported that the specificity of responses to drugs such as apomorphine [26], cocaine [27] and serotonin [28] depends on familiarity with the test environment. This emphasizes the need for evaluation of responses to drugs or stimuli under both challenging and baseline conditions. Removal of the artificial distinction between housing and testing situations is the logic answer. This has led to testing in the permanent housing situations. These home cages must provide the necessary stimuli for specific testing which are usually overlapping with the necessary stimuli for the display of essential species-specific behaviours [29]. Together they could be characterized as enrichment although this term is usually not very well defined.

Complexity of the test environment has an additional function apart from providing the relevant stimuli for testing. If all kind of environmental laboratory conditions are hard to standardize, their influence can be reduced to background noise by enhancing sensory input of relevant stimuli presented to the animals in their cage [7]. It is like providing background radio in animal facilities to diminish the influence of human conversation as is often practised. Thus, an enriched standardized home cage might diminish some influences of the environment to background noise.

\section{Inevitable automation of behavioural observations and analysis}

It has always been recognized, especially in genomics, that the disadvantage of intensive labour and data analysis problems

\section{Table I. Requirements of the 'ideal behavioural test'}

- measure both novelty-induced and baseline behaviors

- address multiple interacting motivational systems

- detect circadian rhythmicity of activity

- minimal human interference

- analyze behaviour as patterns

- recognize visual and acoustical displays

- implements programmable presence of specific stimuli

- contains a minimum of stimuli allowing species specific behaviours

- integrates behavioural observations with physiological measurements should be addressed by automation. In fact, biology in general is undergoing a revolution because of the increasing volume of data especially in fields, like ecology and behaviour, which require a more holistic approach rather than reductionistic.

Automation of behavioural tests started with the use of electro-mechanical devices for experimental control when specific action-reaction or stimulus-response relations had to be quantified. This is particularly useful in a Skinner-box type of setting. Infra red beams were used in all kind of test arenas to monitor activity. These technical developments led to the emergence of batteries of highly automated 'standard' tests, such as PsychoScreen ${ }^{\mathrm{TM}}$ (http://www.psychogenics.com/) and SHIRPA [30]. Such technical modifications all fit in the rationale of answering straightforward questions while focusing on a limited set of stimuli and responses.

In the area of functional studies with a more ethological rationale, automation of complex test environments is well underway. The automated analysis of computer-captured video images in behaviour is known since decades [31]. In fact what we now see is a merge of Skinner box-like technology and video imaging implemented in an enriched home cage. There are several developments in this area (Table 2). The technologies summarized vary in recording methods from video images to body temperature sensors and range from rather basic with regard to the number of motivational systems that are addressed (HomeCageScan, LABORAS, MLog, LabMaster and Home Cage Video Tracking System) to more complex (SmartCube, IntelliCage and PhenoTyper; Fig. 1).

The IntelliCage by NewBehavior is designed to mimic some aspects of a natural situation in which group-housed mice can be monitored for a longer time. It allows the automated measurement of complex learning behaviour in the home cage. The willingness to learn, learning ability, individual competition for food and so forth can all be studied. It is a sophisticated design of which the analysis of the data - to take full advantage of its possibilities - undoubtedly requires equally sophisticated approaches. The complicated, vulnerable and expensive hardware and the transponder-based identification method preventing the distinction of body posture and orientation are disadvantages. PhenoTyper from Noldus Information Technology leans on video tracking for detailed information on position and infrared illumination to allow $24 \mathrm{~h}$ recordings independent of changing ambient light conditions. The system is equipped with stimuli such as an aversive light spot to elicit a diverse range of behaviours. Disadvantage is the solitary housing requirement. Furthermore, although, the video-based recording method is suitable for automated recognition of body postures to detect behaviours like grooming and rearing, this is not yet available. The Smart Vivarium, although still under development in a joint project by the University of California, San Diego and the UC Irvine, is focussing on this gap in behaviour recognition algorithms. Another important advantage of this system, 


\begin{tabular}{|c|c|c|c|c|c|}
\hline System & Available? $^{\mathrm{a}}$ & Company/institute & Pros & Cons & References $^{\mathrm{b}}$ \\
\hline SmartCube & NO & $\begin{array}{l}\text { Psychogenics } \\
\text { www.psychogenics.com }\end{array}$ & $\begin{array}{l}\text { Video-based system detects broad } \\
\text { spectrum of motivational systems and } \\
\text { behaviour, built-in pharmacological database }\end{array}$ & Not available, no validation/test reports & $N A^{c}$ \\
\hline IntelliCage & YES & $\begin{array}{l}\text { NewBehavior } \\
\text { www.newbehavior.com/products/ic }\end{array}$ & $\begin{array}{l}\text { Transponder-based identification of } \\
\text { group housed mice, sophisticated devices } \\
\text { for spatial and temporal learning }\end{array}$ & $\begin{array}{l}\text { No video-processing possible, } \\
\text { no behaviour recognition }\end{array}$ & [32] \\
\hline PhenoTyper & YES & $\begin{array}{l}\text { Noldus Information Technology } \\
\text { www.noldus.com }\end{array}$ & $\begin{array}{l}\text { Video-based tracking of movement patterns, } \\
\text { built-in stimuli to detect avoidance behaviour, } \\
\text { operant conditioning }\end{array}$ & $\begin{array}{l}\text { Solitary housing, limited behaviour } \\
\text { recognition }\end{array}$ & {$[33,34]$} \\
\hline Smart Vivarium & NO & University of California, San Diego \& UC Irvine & $\begin{array}{l}\text { Focus on intelligent algorithms for behaviour } \\
\text { recognition, existing lab cages can be used, } \\
\text { social housing }\end{array}$ & $\begin{array}{l}\text { Limited motivational systems addressed, } \\
\text { not available, no validation/test reports }\end{array}$ & [35] \\
\hline HomeCageScan & YES & $\begin{array}{l}\text { Clever Sys Inc. } \\
\text { www.cleversysinc.com/CSIHome.html }\end{array}$ & $\begin{array}{l}\text { Video-based system, extensive behaviour } \\
\text { recognition, existing lab cages can be used }\end{array}$ & $\begin{array}{l}\text { Solitary housing, limited motivational } \\
\text { systems addressed }\end{array}$ & NA \\
\hline LABORAS & YES & $\begin{array}{l}\text { Metris } \\
\text { www.metris.n//laboras }\end{array}$ & $\begin{array}{l}\text { Monitoring based on vibration and force } \\
\text { signal analysis, existing lab cages can be used, } \\
\text { behaviour recognition }\end{array}$ & $\begin{array}{l}\text { Solitary housing, setup highly sensitive to } \\
\text { physical environment, limited motivational } \\
\text { systems addressed }\end{array}$ & {$[36,37]$} \\
\hline $\begin{array}{r}\text { Home Cage Video } \\
\text { Tracking System }\end{array}$ & YES & $\begin{array}{l}\text { Med Associates } \\
\text { www.med-associates.com/new_prod/video.htm }\end{array}$ & $\begin{array}{l}\text { Video-based tracking of movement patterns, } \\
\text { cages in sound attenuating cubicle }\end{array}$ & $\begin{array}{l}\text { Solitary housing, no behaviour recognitions, } \\
\text { limited motivational systems addressed }\end{array}$ & NA \\
\hline MLog & YES & $\begin{array}{l}\text { Biobserve } \\
\text { www.biobserve.com }\end{array}$ & $\begin{array}{l}\text { Recording of activity by passive infrared } \\
\text { detectors, real time data analysis and remote } \\
\text { control, existing cages can be used }\end{array}$ & $\begin{array}{l}\text { Solitary housing, no behaviour recognition, } \\
\text { very limited variety of measurements possible } \\
\text { (only activity vs. no activity) }\end{array}$ & NA \\
\hline LabMaster & YES & $\begin{array}{l}\text { TSE } \\
\text { www.tse-systems.com/labmaster/labmaster.htm }\end{array}$ & $\begin{array}{l}\text { Photo-beam system for monitoring both } \\
\text { home cage activity and metabolic performance }\end{array}$ & $\begin{array}{l}\text { Solitary housing, no behaviour recognition, } \\
\text { limited motivational systems addressed }\end{array}$ & NA \\
\hline
\end{tabular}

${ }^{a}$ Whether system is commercially available for customers.

${ }^{\mathrm{b}}$ Publication in peer-reviewed journal.

${ }^{\mathrm{C}}$ Not available. 
(a)

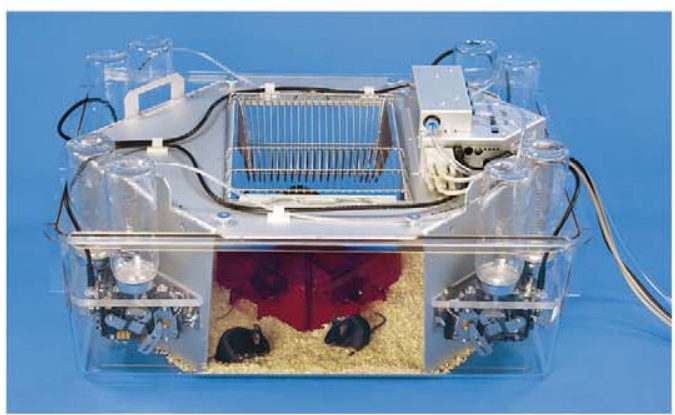

(b)

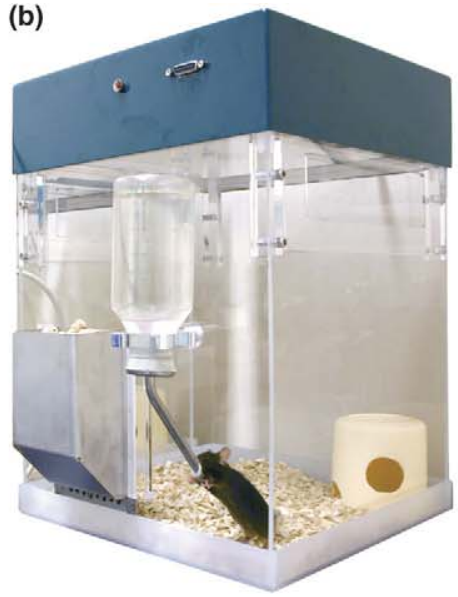

(c)

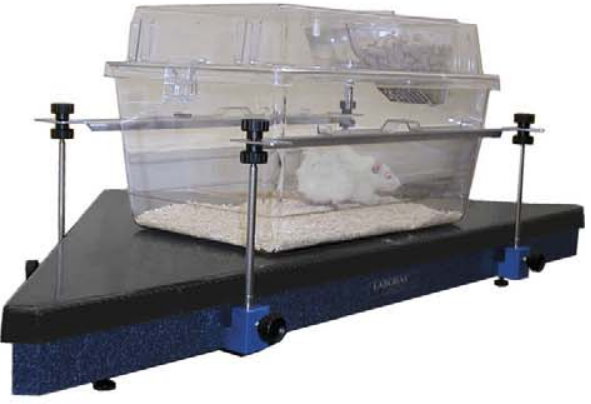

Figure I. Examples of automated home cage observation systems: (a) IntelliCage, (b) PhenoTyper and (c) LABORAS

which also holds for, for example, the LABORAS system by Metris, is that existing lab cages can be used.

Monitoring continuous visual and acoustic displays as an ongoing stream of events requires continuous processing of digitized video images and sound. Detailed recordings of position alone yield already an enormous set of data. The characteristics of movement patterns concern numerous parameters, shape, angularity, stops, walking bouts, velocity and distance moved calculated for each zone and time unit. Recordings of a few days with an analysis per hour yield an excessive number of parameters. Especially when events are programmed and the animal's reactions have to be analysed in relation to such an event.

If variability in human observation of behaviour has been a disadvantage, especially in more complicated tasks, then this is improved by replacement of the human eye, translating the implicitly learned observing techniques into explicitly formulated algorithms that can be employed by an intelligent device. IntelliCage and PhenoTyper are both good examples of such a new development. Both have also the indispensable possibility to integrate behavioural parameters with physiological parameters as measured by telemetric transmitters or transponders: heart rate, blood pressure and temperature. Now what the use of a computer's eye and other integrated sensors demonstrate, are the limitations of the human senses to quantify data and its brain to process such vast complicated sets of data. It seems trivial but the limiting factor is not the collection of integrated data and continuous streams of displays and sounds but the tools to process them.

\section{Standardization problem reappearing}

Lack of inter-observer reliability has always been a major concern when comparing data across laboratories. Now with the rise of novel techniques for the automated analysis of behaviour this problem is reappearing on a different level; how to compare data obtained by different analysing techniques, for example vibration versus image processing? Each manufacturer of automated systems is now using its own validation criteria and material as input for the algorithms that should be able to recognize individual behaviours such as grooming and rearing. However, as researchers will increasingly use automated systems for their behavioural assays, a new challenge emerges. Ideally, we should have an internationally accepted ethogram of rodent behaviour containing definitions and accompanying video material. An expert committee of researchers working in behavioural science should be appointed to evaluate the video material and define the individual behaviours. This database then, will be the basis for validation of algorithms developed by manufacturers of automated analysis systems.

Thus, well-defined ethograms as input for automation: the human observer is inconsequent and definitions of behaviour in ethogram are interpreted in various ways. A computer algorithm, however, once programmed and trained is consistent and unbiased. This emphasizes the importance of putting effort and time in providing the algorithms with the right input to validate them. The scientific community is responsible for the establishment of this 'golden standard' of rodent behaviours and it should be demanded from manufactures to use it for the development of their automated systems.

\section{Conclusion}

The first steps in automation might, thus, have overcome the time-consuming efforts of human observers and the development of testing in home cage situations has the potential to overcome confounding factors such as environmental variation and human interference. But until now, the issue of obtaining and analysing the appropriate data for behaviour 
recognition and pattern analysis is still not properly addressed.

Behaviour, like language, has a grammar and words; it consists of a series of sequential functionally related events and requires analysis of patterns [38] next to frequencies and durations. It is contra intuitive that an advanced analysis of behavioural data has not fully taken advantage of sophisticated analysis techniques (sequential- or cluster analysis) data mining algorithms, and so forth. This point has been addressed by Gerlai [4] who calls 'bioinformatics to the rescue'; monitoring brain function, be it at the level of behaviour or electrophysiology, requires a mathematical approach.

What is lacking is a smart interactive interface between the data and the scientist who wants to explore its data in a sophisticated way. Another weak point is image recognition: The computer's eye and the human visual cortex still process information differently. Computerized systems have a magnificent performance in movement detection but as yet a poor performance in behaviour recognition in freely moving animals. What we need here is artificial intelligence to equip computers with improved abilities to recognize forms and to endow them with the ability to be trained according standard video clips or photographs, which are agreed upon by a scientific community of experts.

The use of artificial neural networks to be used in connection with a set of digitized video images has been validated before showing high reliability [39].

$\mathrm{Al}$ technology has been implemented in other areas such as genomics [40] and it seems too little and rather late in animal behaviour sciences.

What can be seen over time and probably is a sensible development is the use of more and more software based tools and less hardware, which is impractical to use in an animal facility and difficult to adapt for other purposes. Sensors necessary to allow computers to monitor behaviour originate often from other areas of expertise, for example in surveillance.

One of the main factors preventing this evolution is not the availability of technology but a psychological one. Behaviour might be recognized as the most complicated product of the brain, at the same time it is something everybody can observe and describe. But the scientific analysis of animal behaviour should be more precise and unbiased by prejudices to elucidate causal mechanisms. We cannot see genes and molecules; thus, it appears self evident that you need instruments and sophisticated data mining and analysis tools to study them. For behaviour we have to accept that even if we intuitively have ideas about it, we also need ethologically driven mathematics and computer science to reliably study, understand and interpret it. Its complexity is highly underestimated which is demonstrated by differences in investments in for instance genomics and phenomics. The paucity of appropriate animal models and the complexity of behavioural phenotyping have become the most limiting factors in

\section{Related articles}

Brunner, D., et al. (2002) In need of high-throughput behavioral systems. Drug Discov. Today 7, 107-SII2

Gerlai, R. (2002) Phenomics: fiction or the future? Trends Neurosci. 25 , 506-509

Tecott, L.H. and Nestler, E.J. (2004) Neurobehavioral assessment in the information age. Nat. Neurosci. 7, 462-466

Wahlsten, D., et al. (2003) In search of a better mouse test. Trends Neurosci. 26, 132-136

Würbel, H. (2002) Behavioral phenotyping enhanced - beyond (environmental) standardization. Genes Brain Behav. I, 3-8

\section{Outstanding issues}

- Integration of measurements of behavioural, acoustical and physiological variables.

- Data-mining tools to handle large and complex sets of data.

- Sophisticated behavioural recognition software.

- International agreement on definitions of individual rodent behaviours.

- Solid validation of complex home cage based experimental set ups.

understanding the adaptive significance of mechanisms underlying behaviour.

\section{References}

1 McClearn, G.E. (2004) Nature and nurture: Interaction and coaction. Am. J. Med. Genet. Part B: Neuropsychiatr. Genet. 124B, 124-130

2 Cooper, R.M. and Zubek, J.P. (1958) Effects of enriched and restricted early environments on the learning ability of bright and dull rats. Can. J. Psychol. $12,159-164$

3 Würbel, H. (2002) Behavioral phenotyping enhanced - beyond (environmental) standardization. Genes Brain Behav. 1, 3-8

4 Gerlai, R. (2002) Phenomics: Fiction or the future? Trends Neurosci. 25, 506-509

5 Wahlsten, D. et al. (2003) In search of a better mouse test. Trends Neurosci. 26, 132-136

6 Brunner, D. et al. (2002) In need of high-throughput behavioral systems. Drug Discov. Today 7 pp. 107-S112

7 Wolfer, D.P. et al. (2004) Laboratory animal welfare cage enrichment and mouse behaviour. Nature 432, 821-822

8 Lathe, R. (2004) The individuality of mice. Genes Brain Behav. 3, 317-327

9 Hendrie, C.A. and Weiss, S.M. (1994) The development of an animal model of panic with predictive and face validity. In Ethology and psychopharmacology (Cooper, S.J. and Hendrie, C.A., eds), pp. 85-109, John Wiley \& Sons Ltd

10 Blanchard, D.C. et al. (2001) Mouse defensive behaviors: Pharmacological and behavioral assays for anxiety and panic. Neurosci. Biobehav. Rev. 25, 205-218

11 van Ree, J.M. and Wolterink, G. (1981) Injection of low doses of apomorphine into the nucleus accumbens of rats reduces locomotor activity. Eur. J. Pharmacol. 72, 107-111

12 Tecott, L.H. and Nestler, E.J. (2004) Neurobehavioral assessment in the information age. Nat. Neurosci. 7, 462-466

13 Crawley, J.N. (2000) What's wrong with my mouse? Behavioural phenotyping of transgenic and knockout mice Wiley-Liss

14 Kopp, C. et al. (1999) Comparative study of emotional behaviour in three inbred strains of mice. Behav. Process. 47, 161-174

15 Griebel, G. et al. (2000) Differences in anxiety-related behaviours and in sensitivity to diazepam in inbred and outbred strains of mice. Psychopharmacology 148, 164-170 
16 Carola, V.et al. (2002) Evaluation of the elevated plus-maze and open-field tests for the assessment of anxiety-related behaviour in inbred mice. Behav. Brain Res. 134, 49-57

17 Trullas, R. and Skolnick, P. (1993) Differences in fear motivated behaviors among inbred mouse strains. Psychopharmacology 111, 323-331

18 Avgustinovich, D.F. et al. (2000) Features of the genetically defined anxiety in mice. Behav. Genet. 30, 101-109

19 Crawley, J.N. et al. (1997) Behavioral phenotypes of inbred mouse strains: Implications and recommendations for molecular studies. Psychopharmacology 132, 107-124

20 Crabbe, J.C. et al. (1999) Genetics of mouse behavior: interactions with laboratory environment. Science 284, 1670-1672

21 Wahlsten, D.M. et al. (2003) Different data from different labs: Lessons form studies of gene-environment interaction. Wiley Periodicals pp. 283-311

22 Rampon, C. et al. (2000) Enrichment induces structural changes and recovery from nonspatial memory deficits in ca1 nmdar1-knockout mice. Nat. Neurosci. 3, 238-244

23 Miczek, K.A. et al. (1989) Temporal and sequential patterns of agonistic behavior: effects of alcohol, anxiolytics and psychomotor stimulants. Psychopharmacology 97, 149-151

24 Mitchell, P.J. (1994) Prediction of antidepressant activity from ethological analysis of agonistic behaviour in rats. In Ethology and psychopharmacology (Cooper, S.J. and Hendrie, C.A., eds), pp. 85-109, John Wiley \& Sons Ltd

25 Rodgers, R.J. and Cole, J.C. (1994) The elevated plus-maze: pharmacology, methodology and ethology. In Ethology and psychopharmacology (Cooper, S.J. and Hendrie, C.A., eds), pp. 85-109, John Wiley \& Sons Ltd

26 Harkin, A. et al. (2000) Test conditions influence the response to a drug challenge in rodents. Pharmacol. Biochem. Behav. 65, 389-398

27 Carey, R.J. et al. (2005) Acute and chronic cocaine behavioral effects in novel versus familiar environments: open-field familiarity differentiates cocaine locomotor stimulant effects from cocaine emotional behavioral effects. Behav. Brain Res. 158, 321-330

28 Joyce, D. and Mrosovsky, N. (1964) Eating, drinking and activity in rats following 5-hydroxytryptophan (5-htp) administration.

Psychopharmacologia 5, 417-423
29 Gerlai, R. and Clayton, N.S. (1999) Analysing hippocampal function in transgenic mice: An ethological perspective. Trends Neurosci. 22, 47-51

30 Rogers, D.C. et al. (1997) Behavioral and functional analysis of mouse phenotype: SHIRPA, a proposed protocol for comprehensive phenotype assessment. Mamm. Genome 8, 711-713

31 Spruijt, B.M. and Gispen, W.H. (1983) Prolonged animal observation by use of digitized videodisplays. Pharmacol. Biochem. Behavior. 19, 765-769

32 Galsworthy, M.J. et al. (2005) A comparison of wild-caught wood mice and bank voles in the intellicage: Assessing exploration, daily activity patterns and place learning paradigms. Behav. Brain Res. 157, 211-217

33 DeVisser, L. et al. (2005) Automated home cage observations as a tool to measure the effects of wheel running on cage floor locomotion. Behav. Brain Res. 160, 382-388

34 DeVisser, L. et al. Novel approach to the behavioural characterization of inbred mice: automated home cage observations. Genes Brain Behav. (in press)

35 Belongie, S. et al. (2005) Monitoring animal behavior in the smart vivarium. 5th International Conference on Methods and Techniques in Behavioral Research, 30 August-2 September, Wageningen, The Netherlands

36 van de Weerd, H.A. et al. (2001) Validation of a new system for the automatic registration of behaviour in mice and rats. Behav. Process. 53, $11-20$

37 Quinn, L.P. et al. (2003) LABORAS ${ }^{\mathrm{TM}}$ : initial pharmacological validation of a system allowing continuous monitoring of laboratory rodent behaviour. J. Neurosc. Methods 130, 83-92

38 Magnusson, M.S. (2000) Discovering hidden time patterns in behavior: Tpatterns and their detection. Behav. Res. Methods Instrum. Comput. 32, 93110

39 Rousseau, J.B. et al. (2000) Classification of rat behavior with an imageprocessing method and a neural network. Behav. Res. Methods Instrum. Comput. 32, 63-71

40 Laghaee, A. et al. (2005) Artificial intelligence and robotics in high throughput post-genomics. Drug Discov. Today 10, 1253-1259 